\title{
FEDSM-ICNMM2010-30743
}

\section{THE IMPORTANCE OF MEAN FREE PATH IN DETERMINING GAS MICRO FLOW BEHAVIOUR}

\author{
Nishanth Dongari \\ Department of Mechanical Engineering \\ University of Strathclyde \\ Glasgow G1 1XJ, UK \\ email: nishanth.dongari@strath.ac.uk
}

\author{
Yonghao Zhang \\ Jason M. Reese * \\ Department of Mechanical Engineering \\ University of Strathclyde \\ Glasgow G1 1XJ, UK \\ email: jason.reese@strath.ac.uk
}

\begin{abstract}
We investigate whether a power-law form of probability distribution function better describes the free paths of dilute gas molecules in a confined system. An effective molecular mean free path model is derived, which allows the mean free path to vary close to bounding surfaces. Our model is compared with molecular dynamics simulation data, and also other classical mean free path models. As gas transport properties can be related to the mean free path through kinetic theory, the Navier-Stokes constitutive relations are then modified and applied to various benchmark test cases. Results for isothermal pressure-driven Poiseuille flows in micro-channels are reported, and we compare our results with conventional hydrodynamic models, solutions of the Boltzmann equation, and experimental data. Our new approach provides good results for mean free path and crosssectional flow velocity profiles up to Knudsen numbers around 1 , and for integral flow parameters such as flow rate and friction factor up to Knudsen number of 10. We discuss some limitations of our power-law model, and point to the way forward for further development.
\end{abstract}

\section{INTRODUCTION}

In microscale gas flows the small system dimensions mean that the non-dimensional Knudsen number, $K n$, defined as the ratio of the molecular mean free path (MFP) of the gas, $\lambda$, to a

\footnotetext{
* Address all correspondence to this author.
}

characteristic flow field dimension, indicates the flows are often rarefied. As $K n$ increases, the behaviour of a gas flow near a solid bounding surface is dominated by the effect of gas moleculesurface interactions. This leads to the formation of a Knudsen layer (KL): a local thermodynamically non-equilibrium region extending $\sim O(\lambda)$ from the surface. Figure 1 is a schematic of the gas velocity profile in the $\mathrm{KL}$ in a shear-driven flow. Although the Navier-Stokes-Fourier (NS) equations with classical velocity-slip and temperature-jump boundary conditions [1] can often adequately predict the flow field outside the KL, they fail to capture the non-linear form of the KL [2].

In the KL, gas molecule-surface collisions are more frequent than gas molecule-molecule collisions, i.e. the gas MFP will effectively be reduced in the KL. It is well-known from the kinetic theory of gases that viscosity and thermal conductivity can be interpreted in terms of the collisions of gas molecules, and of the free paths of the molecules between collisions [3]. Linear constitutive relations for shear stress and heat flux, with constant thermophysical transport coefficients such as viscosity and thermal conductivity, are not necessarily valid in the KL.

Lockerby et al. [4] and O'Hare et al. [5] developed walldistance scaling models for the constitutive relationship for shear stress in planar flows. The applicability of these models is limited to relatively low- $K n$ flows. Several other scaling functions for rarefied gas flows have been proposed recently: $K n$-dependent functions [6], and power-law scaling of constitutive relations [7, 8]. Both of these latter predict an infinite shear-rate at the wall, 


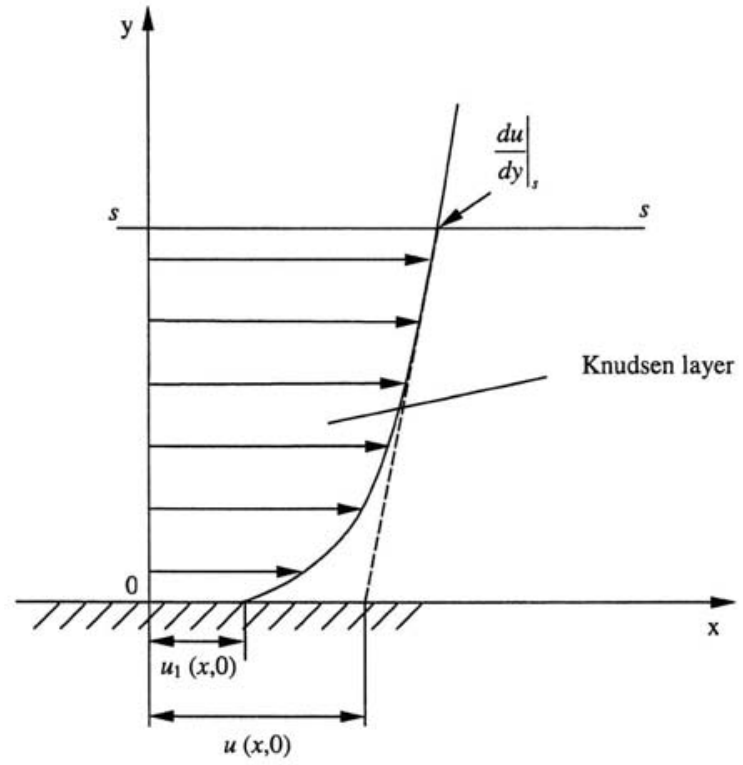

FIGURE 1. Schematic of the momentum Knudsen layer (KL) close to a planar wall, showing the microscopic slip $u_{1}(x, 0)$; the macroscopic slip $u(x, 0)$ is required when using the classical Navier-Stokes equations with a slip boundary condition.

and therefore a zero effective viscosity, which makes numerical results dependent on grid spatial resolution.

Stops [9] derived an effective MFP, $\lambda_{\text {eff(S), which is }}$ geometry-dependent. He investigated the classical probability distribution function, $\psi(r)=\lambda^{-1} \exp (-r / \lambda)$, for which the probability that a given gas molecule will travel a distance in the range $r$ to $r+d r$ between two successive collisions is $\psi(r) d r$. If the gas is not bounded, the MFP of the gas molecule ensemble is simply $\int_{0}^{\infty} N r \psi(r) d r / N=\lambda$, where $N$ is the total number of molecules. If a solid bounding surface is included in the system, however, some molecules will hit the surface and their free flight paths will be terminated. The MFP of all the gas molecules in the system will therefore be smaller than $\lambda$, due to this boundary limiting effect. Recently, Arlemark et al. [10] developed a probability function-based effective MFP expression $\lambda_{\text {eff(A) }}$, also using the classical exponential form of the distribution function. Guo et al. [11] implemented the effective MFP expression derived by Stops [9] in conjunction with a second-order slip boundary condition and obtained good results for pressure-driven rarefied gas flows.

However, comparison of both of these effective MFP profiles with molecular dynamics (MD) simulation data [12] shows that both models are accurate only up to Knudsen numbers of about 0.2. The MD data [12] highlights the limitations of the exponential form of probability distribution function for gases in the transition regime, as it can only provide an accurate de- scription of a gas under thermodynamic equilibrium [9]. Anomalous diffusive transport is often better described using Lévy and power-law (PL) probability distributions $[13,14]$. Anomalous transport can be characterised by incorporating a spatial dependence in the diffusion coefficient, $D$; for example, $D(x) \propto$ $|x|^{-n}$ [13]. For ideal gases, $D=(1 / 3) \bar{v} \lambda$ and $\rho D=\mu$, with $\bar{v}$ being the molecular mean velocity, $\mu$ the dynamic viscosity of the gas and $\rho$ the density of the gas [15]. In anomalous gas transport, the MFP would be spatially dependent and may take a PL form. This kind of diffusion coefficient has been applied to several physical situations, such as fast electrons in a hot plasma in the presence of a DC electric field, turbulent two-particle diffusion in configuration space [16] and mean first passage time for anomalous diffusive systems [13].

This paper investigates the hypothesis that the anomalous transport behaviour of non-equilibrium gases is better understood by using PL type of distribution functions. We derive an effective MFP based on a PL probability distribution of free paths for a gas close to a planar wall, and show that this captures some of the trends associated with the complex non-equilibrium physics of transition regime gas flows. Our PL-based effective MFP is validated against MD simulation data [12] up to $K n=2$ and also compared with the theoretical models of Stops [9] and Arlemark et al. [10].

\section{AN EFFECTIVE MOLECULAR MEAN FREE PATH Equilibrium mean free path}

The individual free path-lengths of gas molecules between intermolecular collisions in a dilute gas vary widely. However, if we follow a molecule until it has traversed a great many free paths, the average of these path-lengths will have a definite value, which is called the mean free path (MFP). In a time $t$ that is long compared to the time of molecular collisions we can write:

$$
\lambda=\frac{\bar{v}}{\dot{\theta}_{v}},
$$

where $\dot{\theta}_{v}$ denotes the collision rate for a molecule of a group moving at speed $\bar{v}$ among molecules composing the gas. The collision rate is the chance per second that a particular molecule collides; it might appropriately also be called the collision probability per unit time, $P_{v}$.

The distribution of the lengths of the individual molecular paths is also of interest. Let us consider a group of similar molecules that are moving with velocity $\bar{v}$ through a region in which there is gas. The number originally in the group at time $t=0$ is $N_{0}$, and by time $t$ let $N$ of them still be travelling without having had a collision. During the following interval $d t, N \dot{\theta}_{v} d t$ molecules will collide and drop out of the group; $N$ is thereby 
changed by the amount:

$$
d N=-N \dot{\theta}_{v} d t
$$

We can easily integrate this equation under thermodynamic equilibrium conditions. By thermodynamic equilibrium we mean that the probability of a certain microscopic state averaged over the details of the interactions, does not change in time or space [18]. So, with $\dot{\theta}_{v}$ constant, $d N / N=-\dot{\theta}_{v} d t$, thus $\ln N=-\dot{\theta}_{v} t+$ const . Choosing the constant of integration so as to make $N=N_{0}$ at $t=$ 0 , we find

$$
N=N_{0} \exp \left(-\dot{\theta}_{v} t\right)=N_{0} \exp \left(-\frac{\dot{\theta}_{v}|r|}{\bar{v}}\right),
$$

where $|r|$ is the length $\bar{v} t$ of free path that has been travelled at time $t$ by each molecule. Let $\varphi(r)$ be the fraction of the original $N_{0}$ molecules that have travelled a distance $r$ without collision, and $\psi(r) d r$ be the fraction of all the free paths that have a length between $r$ and $r+d r$. Then $\varphi(r)=N / N_{0}$ and $\psi(r) d r=d N / N_{0}$, hence from Eq. (1)

$$
\varphi(r)=\exp \left(-\frac{|r|}{\lambda}\right), \psi(r)=\frac{1}{\lambda} \exp \left(-\frac{|r|}{\lambda}\right) .
$$

So $\psi(r)$, the probability distribution of free paths, is only exponential in form under thermodynamic equilibrium conditions and when the gas is unbounded. If the MFP, calculated for a molecule in an extensive material, is comparable with a linear dimension of the finite system confining the gas, the boundaries of the system may limit the effective free-path values. However, Stops [9] and Arlemark et al. [10] used the exponential form of the distribution function, Eq. (4), to derive effective MFP models as a function of distance from a bounding surface.

\section{A model for non-equilibrium mean free path}

In a rarefied gas system, in which gas molecules may not suffer sufficiently frequent collisions with other gas molecules to attain equilibrium conditions, deviations from thermodynamic equilibrium may have substantial effects. Although it is certainly difficult to evaluate the probability distribution function $\psi(r)$, introducing non-equilibrium into Eq. (2) by means of physical arguments could allow us to propose a distribution function that may be more appropriate for non-equilibrium conditions than the exponential one [18].

Montroll \& Scher [14] pointed out that a finite moment of the probability distribution function implies an exponential character of the randomness. So results obtained using exponential forms of the distribution functions are essentially the same as those for homogeneous media at equilibrium. A distribution function with diverging higher-order moments, such as the standard deviation, is essential to anomalous transport. In transient photoconductivity experiments, concentration distributions may have a long tail such that the standard deviation is diverging. This long-tail problem is common to various flows in non-uniform media, in many fields such as chemical engineering and environmental sciences [19].

We hypothesize a PL form for the probability distribution function for non-equilibrium MFP, with diverging higher-order moments, instead of the classical exponential form of distribution function. Here we propose for investigation the following form of molecular free path distribution function:

$$
\psi(r)=C(a+r)^{-n},
$$

where $a$ and $C$ are constants with positive values determined through the zero and first moments. The range of values for the exponent $n$ can be obtained by making one of the higher-order moments divergent. Zero and first moments are given as follows:

$$
1=\int_{0}^{\infty} C(a+r)^{-n} d r
$$

$$
\lambda=\int_{0}^{\infty} C r(a+r)^{-n} d r .
$$

Equation (6) requires the probability to range only from zero to one. Equation (7) defines the unconfined, conventional MFP value, $\lambda$. It then follows that

$$
C=(n-1) a^{n-1},
$$

$$
a=\lambda(n-2) .
$$

If $n>2, a>0$, otherwise the distribution function is negative. The standard deviation (second moment) of the distribution function given by Eq. (5) diverges only for $n \leq 3$; so $2<n \leq 3$. If one wishes to make the $i^{\text {th }}$ moment diverging, then $\max (n)=i+1$. As $n \rightarrow \infty$, the distribution function will have finite moments, which is the condition required of an equilibrium distribution function. For a finite $n$, the distribution function describes a system deviating from equilibrium. Thus, $n$ acts as a decisive parameter to define the extent of deviation from equilibrium. Here, we test $n=3$, unless otherwise explicitly stated. 


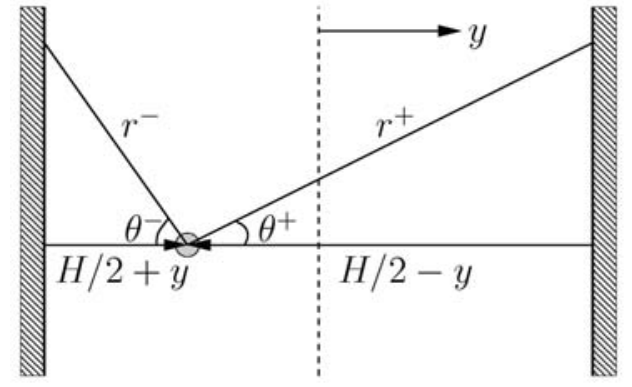

FIGURE 2. A molecule confined between two planar walls a distance $H$ apart. The molecule has an equal probability to travel in any zenith angle $\theta^{-}$or $\theta^{+}$or to travel in either the positive or negative $y$-direction. The molecule under consideration is assumed to have just experienced an intermolecular collision at its current position $H / 2+y$ above the lefthand surface.

We follow the approach of Arlemark et al. [10], based on an integrated form of the probability distribution function, i.e.

$$
p(r)=\int_{r}^{\infty} \psi(r) d r=\left[1-\left(1+\frac{r}{a}\right)^{1-n}\right]
$$

which describes the probability a molecule travels a distance $r$ without experiencing a collision.

Our model is derived for the two-planar-wall configuration shown in Fig. 2. We use the notation $r^{-}$if a test molecule is travelling in the negative $y$-direction, and $r^{+}$if the molecule is travelling in the positive $y$-direction. We also use the notations $\theta^{-}$and $\theta^{+}$for the equally probable zenith angle travelling direction of the molecule. These quantities are related through $r^{-}=(H / 2+y) / \cos \theta^{-}$and $r^{+}=(H / 2-y) / \cos \theta^{+}$, where $H$ is the distance between the two parallel plates.

The MFP based on the PL form of distribution function, $\lambda_{\text {eff(PL) }}$, is expressed by weighting the unconfined MFP, $\lambda$, with $p(r)$ as follows:

$$
\begin{aligned}
\lambda_{\mathrm{eff}(\mathrm{PL})} & =\frac{\lambda}{2}\left[p\left(r^{-}\right)+p\left(r^{+}\right)\right] \\
& =\lambda\left\{1-\frac{1}{2}\left[\left(1+\frac{r^{-}}{a}\right)^{1-n}+\left(1+\frac{r^{+}}{a}\right)^{1-n}\right]\right\} .
\end{aligned}
$$

A 3-dimensional MFP depending on the gas molecule's distance to a surface is then obtained by averaging the free path with respect to $\theta^{-}$and $\theta^{+}$in the range $[0, \pi / 2]$ using the mean integral theorem,

$$
<\lambda_{\mathrm{eff}(\mathrm{PL})}(\theta)>=\frac{2}{\pi} \int_{0}^{\pi / 2} \lambda_{\mathrm{eff}(\mathrm{PL})}(\theta) d \theta
$$

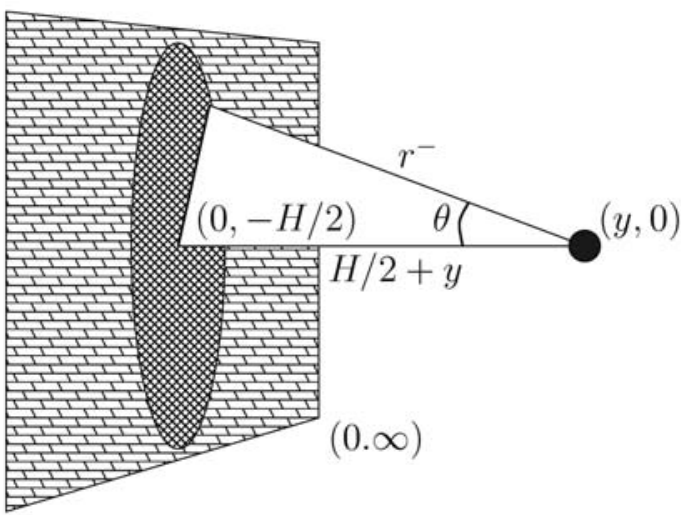

FIGURE 3. A molecule at a distance $H / 2+y$ from a planar wall; possible trajectories for travelling in the negative $y$-direction in cylindrical coordinates $[H / 2+y,(H / 2+y) \tan \theta]$.

where the integration domain is illustrated in Fig. 3 for a gas molecule travelling in the negative $y$-direction. Averaging over the free path in Eq. (12), using Simpson's numerical integration involving 16 subintervals, results in $\lambda_{\text {eff(PL) }}=\lambda \beta_{\mathrm{PL}}$, where

$$
\begin{aligned}
\beta_{\mathrm{PL}}=1-\frac{1}{94}[ & \left(1+\frac{H / 2-y}{a}\right)^{1-n}+\left(1+\frac{H / 2+y}{a}\right)^{1-n} \\
& +4 \sum_{i=1}^{8}\left(1+\frac{H / 2-y}{a \cos [(2 i-1) \pi / 32]}\right)^{1-n} \\
& +4 \sum_{i=1}^{8}\left(1+\frac{H / 2+y}{a \cos [(2 i-1) \pi / 32]}\right)^{1-n} \\
& +2 \sum_{i=1}^{7}\left(1+\frac{H / 2-y}{a \cos [i \pi / 16]}\right)^{1-n} \\
& \left.+2 \sum_{i=1}^{7}\left(1+\frac{H / 2+y}{a \cos [i \pi / 16]}\right)^{1-n}\right]
\end{aligned}
$$

which is the normalized effective MFP based on the power-law distribution function, and is evaluated using the rarefaction parameter $K n$ (as $a$ is dependent on mean free path). From Eq. (13), it is easy to see that our PL model satisfies the physically intuitive requirements for $K n \rightarrow 0$, i.e.

$$
\left.\beta_{\mathrm{PL}}\right|_{\text {wall }} \approx 1 / 2, \text { and }\left.\beta_{\mathrm{PL}}\right|_{\text {bulk }} \approx 1
$$

\section{Comparison with Molecular Dynamics simulations}

The $\beta$-function of Eq. (13) is now compared with MD simulation results [12] and the classical exponential probability function models of Stops [9] and Arlemark et al. [10], for both single- 

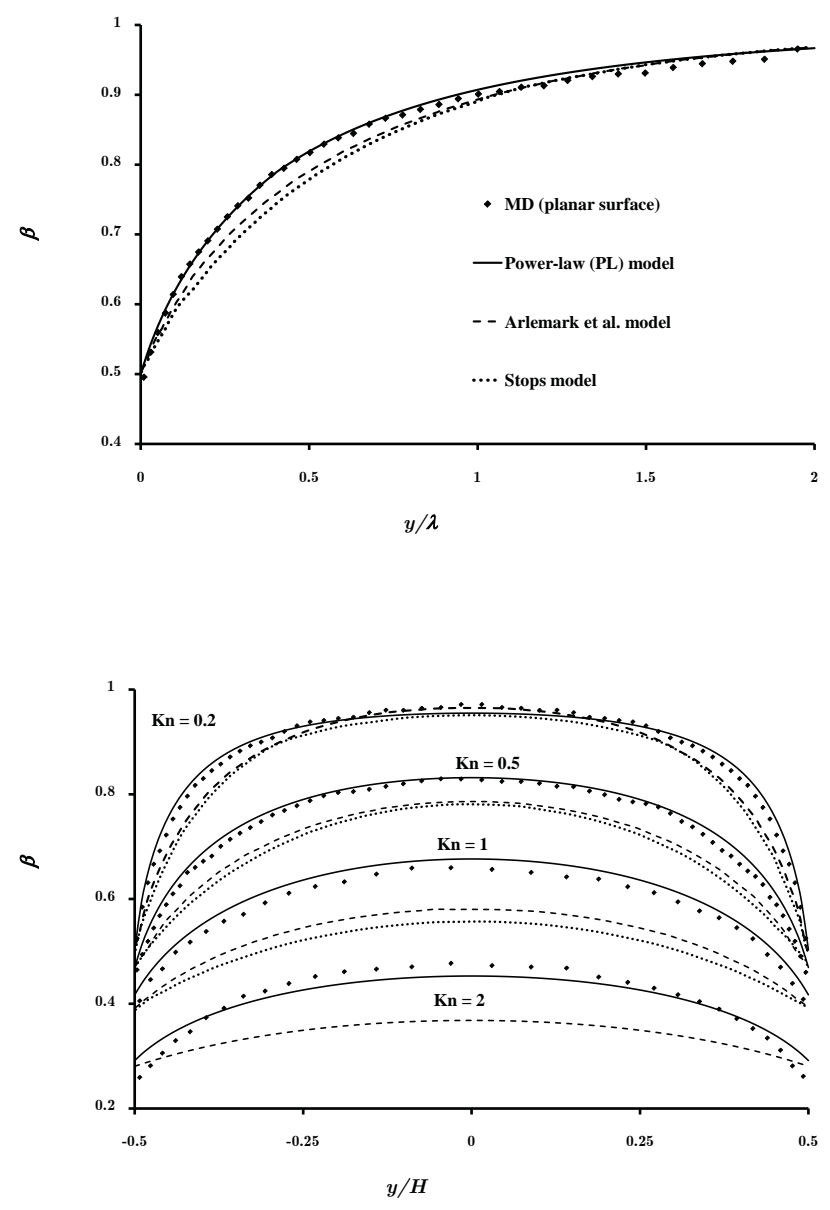

FIGURE 4. Variation of normalized mean free path, $\beta$, with normalized distance from a surface; (a) single-wall case and (b) parallel-wall case. Comparison of power-law (PL) model with molecular dynamics (MD) simulation data [12], and Arlemark et al. [10] and Stops [9] exponential models, for various Knudsen numbers.

and parallel-wall cases. Single-wall results are deduced by assuming that the second wall is located an infinite distance from the first. In the case of parallel walls, KL overlap becomes apparent as $K n$ increases. Arlemark \& Reese [12] performed MD simulations using 20520 Lennard-Jones molecules, yielding results that are estimated to be within 1\% accuracy. All simulations were carried out assuming a planar smooth bounding surface.

Figure 4a shows the variation of normalized effective MFP profiles (i.e. $\beta$ ) with normalized wall distance $y / \lambda$. Our PL model predictions are in close agreement with MD simulations, although minor overpredictions are noticed in the bulk region. The PL model has a sharp gradient close to the wall, while both of the previous exponential-based models have shallower gradients and underpredict the MFP values in the wall vicinity. All three theoretical models converge to a similar value in the bulk region as expected.

Results for the normalized MFP between two parallel surfaces are presented in Fig. 4b. MD simulation results for planar smooth surfaces are compared with both PL and exponential distribution function-based models for various $K n$ in the transition regime. At $K n=0.2$, which is just beyond the slip-flow regime, the PL model is in fair agreement with the MD data in the nearwall region but deviates slightly in the bulk region. The two exponential based models underpredict in the KL, but Arlemark et al. [10] achieve good agreement with the MD data in the bulk region. As the value of $K n$ increases, and the flow becomes increasingly transition-continuum, the classical models fail to predict the effective MFP in the wall region, as well as in the bulk. Both classical models underpredict the effective MFP values, although the Arlemark et al. [10] results are slightly above Stops'. Effective MFP values predicted by the PL model compare very well to the MD data for both $K n=0.5$ and 1, although it overpredicts for $K n=1$ in the bulk. By $K n=2$, when both the Knudsen layers completely overlap each other, the PL model shows significant deviations from the simulation data and overpredicts in the near-wall region, although there is fair agreement in the bulk region. The MD data shows a relatively sharp gradient of effective MFP in the near-wall region, compared to the theoretical predictions.

\section{ISOTHERMAL PRESSURE-DRIVEN RAREFIED GAS FLOWS Problem description and governing equations}

To further test the merits of our PL-based MFP scaling, we consider isothermal pressure-driven gas flow along a planar-wall channel with walls a distance $H$ apart, as depicted in Fig. 2. The flow is assumed to be fully hydrodynamically developed and two-dimensional, isothermal, laminar and steady, with a low Reynolds number $(R e)$ so that inertial effects may be neglected. With these assumptions, the governing equation is:

$$
0=-\frac{\partial P}{\partial x}-\frac{\partial \tau}{\partial y}
$$

where $x$ is the streamwise coordinate, $y$ the wall-normal coordinate, $P$ the pressure and $\tau$ the stress, which is given as:

$$
\tau=-\mu\left[\nabla \mathbf{U}+(\nabla \mathbf{U})^{t r}\right]+\left(\frac{2}{3} \mu-\kappa\right)(\nabla \cdot \mathbf{U}) I,
$$

where $\mu$ is the fluid dynamic viscosity, $\kappa$ the bulk viscosity, $I$ the identity tensor and $t r$ the transpose operator. 
In Eq. (16) we can neglect volume dilation effects for rarefied gases [15]. The collision time, or equivalently the free flight path between two successive collisions of a gas molecule, is closely related to the momentum exchange. The unconfined MFP is related to the shear viscosity [20]:

$$
\mu=\rho \frac{\lambda}{\sqrt{\pi / 2 R T}}
$$

where $R$ is the specific gas constant and $T$ the gas temperature.

Equation (17) is assumed to be valid only for flows that are quasi-equilibrium. As discussed above, within the KL the flight paths of gas molecules are affected by the presence of a solid wall. If we wish to use Eq. (17), we need to take into account the MFP affected by gas molecular collisions with surfaces. If the unconfined expression for the MFP, $\lambda$, is replaced by our effective and geometry-dependent mean free path, $\lambda_{\text {eff((PL) }}$, we obtain a non-constant, geometry-dependent, effective viscosity, $\mu_{\text {eff(PL), }}$ that can then be used to postulate a non-linear stress/strain-rate relation:

$$
\tau=-\underbrace{\frac{\partial U_{x}}{\partial y},}_{\mu_{\mathrm{eff}(\mathrm{PL})}^{\mu} \beta_{\mathrm{PL}}}
$$

where $U_{x}$ is the fluid velocity in the axial direction.

Using Eq. (18) in Eq. (15) results in the modified governing equation:

$$
\mu \frac{\partial}{\partial y}\left(\beta_{\mathrm{PL}} \frac{\partial U_{x}}{\partial y}\right)=\frac{\partial P}{\partial x} .
$$

This needs to be solved in conjunction with an appropriate slip boundary condition to capture the non-equilibrium phenomena in the slip and transition flow regimes.

\section{Slip boundary condition}

Experimental investigations, e.g. [21], have confirmed that the NS equations with the no-slip boundary condition underpredict mass flow rates. Several authors have addressed this problem by employing the Maxwell slip boundary condition [1], which, for a stationary planar wall and an isothermal flow is:

$$
U_{\text {slip }}=-\frac{2-\sigma}{\sigma} \lambda\left(\frac{\partial U_{x}}{\partial y}\right)_{w}
$$

where $U_{\text {slip }}$ is the slip velocity at the fluid-surface interface, $\sigma$ is the tangential momentum accommodation coefficient (TMAC), i.e. the proportion of incident molecules reflected diffusely from the surface, and subscript $w$ are quantities evaluated at the surface.

The first-order slip boundary condition (20) fails to predict the Knudsen-minimum in the mass flow rate [22], and researchers subsequently proposed higher-order slip models for a wide range of Knudsen numbers. A review of a number of these higher-order slip models can be found in Reese \& Zhang [23]. Deissler [24] derived a second-order slip boundary condition based on the concept of MFP for momentum and energy transfer, which can be expressed in its generalized form as:

$$
U_{\text {slip }}=-C_{1} \lambda\left(\frac{\partial U_{x}}{\partial y}\right)_{w}-C_{2} \lambda^{2}\left(\frac{\partial^{2} U_{x}}{\partial y^{2}}\right)_{w} .
$$

While there is no general consensus on the slip coefficients $C_{1}$ and $C_{2}, C_{1}$ is set to $\sim 1$ by many authors and $C_{2}$ has a wide range from -0.5 to $5 \pi / 12$. Cercignani [25] proposed $C_{2}$ to be 0.9756 from kinetic theory considerations, and Hadjiconstantinou [26] corrected $C_{2}$ to 0.31 .

It is important to note that Maxwell [1] and Deissler [24] derived first- and second-order slip boundary conditions assuming that the MFP is constant in the wall-adjacent gas layer. Guo $e t$ al. [11] proposed the following heuristic slip boundary condition to take into account the KL correction:

$$
U_{\text {slip }}=-C_{1}\left(\lambda_{\text {eff }} \frac{\partial U_{x}}{\partial y}\right)_{w}-C_{2}\left[\lambda_{\text {eff }} \frac{\partial}{\partial y}\left(\lambda_{\text {eff }} \frac{\partial U_{x}}{\partial y}\right)\right]_{w} .
$$

This simply reduces to the conventional second-order slip boundary condition (21) if there is no KL, i.e. if $\lambda_{\text {eff }}=$ const . In the present analysis, we implement this second-order slip boundary condition, and use $\lambda_{\text {eff(PL) }}$ given through Eq. (13). Values of $C_{1}$ and $C_{2}$ are chosen as 1 and 0.31 [26] respectively for the results presented here.

\section{Solution procedure}

We require results for the flow velocity profiles across the channel and some integral flow parameters, through solution of the governing equation with the slip boundary condition discussed above. Besides the governing Eq. (19) and slip boundary condition (22), the ideal gas law, $P=\rho R T$, and the Knudsen number definition $K n=\lambda / H$, will be used in the analysis.

The wall-normal coordinate $y$ is normalized by $H$, and the axial velocity $U_{x}$ by the free-molecular velocity $U_{0}=$ $-2 H(\partial P / \partial x) /(\rho \sqrt{2 R T})$ [27]. Using Eqs. (17) and (13), Eq. (19) in normalized form (indicated by “*”) is then

$$
\frac{\partial}{\partial y^{*}}\left(\beta_{\mathrm{PL}} \frac{\partial U^{*}}{\partial y^{*}}\right)=-\frac{\sqrt{\pi}}{2 K n}
$$



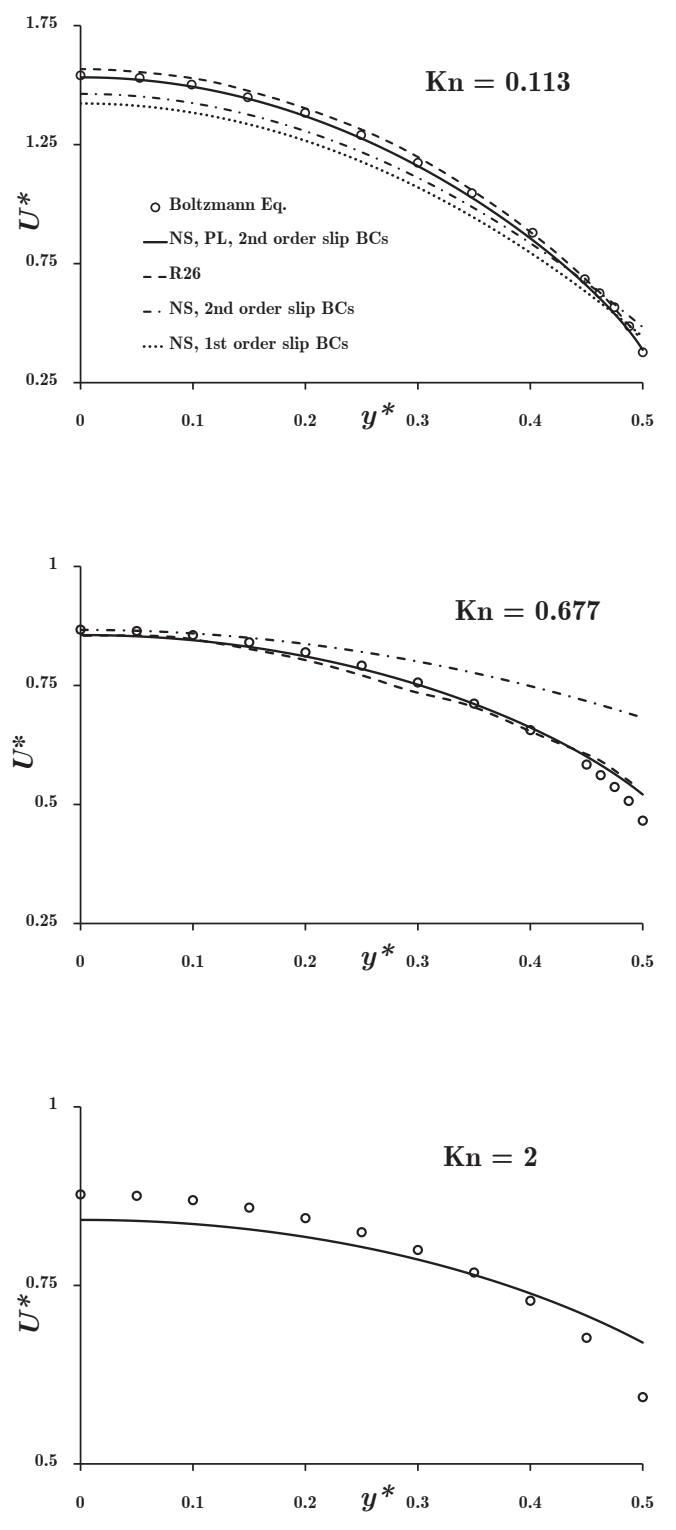

FIGURE 5. Normalized half-channel velocity profiles for various Knudsen numbers. Comparison of power-law (PL) model results with the solution of the Boltzmann equation [27], R26 moment equations [28] and conventional NS equations with first- and second-order slip.

and Eq. (22) in normalized form as

$$
\begin{aligned}
\left(U^{*}\right)_{\text {slip }}= & -C_{1} K n\left(\beta_{\mathrm{PL}} \frac{\partial U^{*}}{\partial y^{*}}\right)_{w} \\
& -C_{2} K n^{2}\left[\beta_{\mathrm{PL}} \frac{\partial}{\partial y^{*}}\left(\beta_{\mathrm{PL}} \frac{\partial U^{*}}{\partial y^{*}}\right)\right]_{w} .
\end{aligned}
$$

Equation (23) is numerically solved for the normalized axial velocity profiles $U^{*}$ by applying the slip boundary condition (24) at the upper wall, $y^{*}=0.5$, and a symmetry condition at the centre of the channel, $y^{*}=0$.

The normalized mass flow rate $G$, an integral flow parameter, is

$$
G=\frac{2 \int_{0}^{H / 2} \rho U_{x} d y}{\rho U_{o} H}=2 \int_{0}^{0.5} U^{*} d y^{*}
$$

\section{RESULTS}

In what follows, we refer to the $P L$ model as the modified governing equation (23), with the use of effective MFP (Eq. 13) and the second-order slip boundary condition (24).

The cross-sectional velocity profiles of pressure-driven Poiseuille flow at a range of Knudsen numbers are presented in Fig. 5. Our PL model is validated with a Boltzmann solution [27] and compared with three hydrodynamic models: conventional first- and second-order slip models, and the R26 equations [28]. At $K n=0.113$, which is just beyond the slip-flow regime, the four hydrodynamic models predict similar values of slip velocity at the wall and all are close to the solution obtained from the Boltzmann equation. The conventional NS equations with both first- and second-order slip boundary conditions significantly underpredict the velocity in the bulk region. The PL model and R26 equations are in fair agreement with the Boltzmann solution, although the PL model slightly underpredicts, and the R26 equations overpredict, the maximum velocity.

At higher $K n$, the flow enters the transition regime and the non-equilibrium regions from both parallel walls start to overlap each other. At $K n=0.677$, the PL model and R26 equations compare reasonably well with the Boltzmann solutions, although the R26 equations overpredict the slip velocity. At $K n=2$, deviations from the Boltzmann predictions are relatively less for our PL model than for the R26 equations.

Predictions of normalized slip velocity, $\left(U^{*}\right)_{\text {slip }}$, are presented as a function of $K n$ in Fig. 6, and compared to Boltzmann simulation data [27]. First-order slip is a constant, and higher than the Boltzmann result for $K n<1$ and lower for $K n>1$. The second-order NS slip is too high, particularly at large Knudsen numbers. Our PL model and the R26 equations agree well with the Boltzmann solution up to $K n \simeq 0.5$. Both models overpredict the wall-slip velocity for $K n>0.5$, although the deviations are relatively less with the PL model.

It is evident from Figs. 4 and 5 that in the transition regime, non-equilibrium effects are no longer limited to the wall-adjacent layer, but prevail in the bulk flow due to the overlap of Knudsen layers. Hence, simply using a higher-order slip model with modified TMAC's may result in good wall-slip velocity results but will not improve the overall predictive capabilities of the NS 


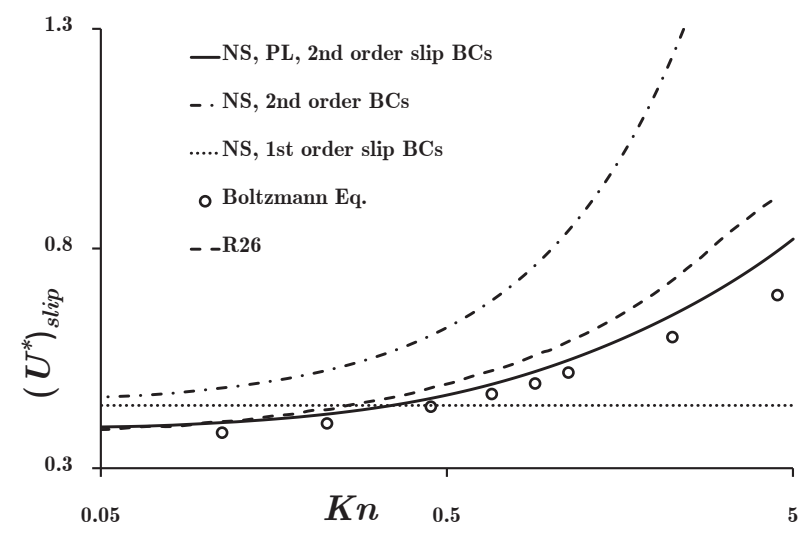

FIGURE 6. Variation of normalized slip velocity with Knudsen number; comparison of power-law (PL) model with the solution of the Boltzmann equation [27], R26 moment equations [28] and the conventional NS equations with first- and second-order slip.

equations in the transition regime. In addition, the accurate prediction of integral flow parameters in micro-conduits is important in engineering MEMS devices. To predict the integral flow parameters correctly, it is essential that field variables such as MFP and cross-sectional velocities are correct. Conversely, however, the accurate prediction of integral flow parameters does not necessarily guarantee that all the field variables have each been predicted accurately.

Figure 7 shows the variation of normalized flow rate $G$ with inverse Knudsen number, $\delta_{m}=\sqrt{\pi} /(2 K n)$. In Fig. 7a, the PL model results are compared with experimental data for different pressure ratios [21], BGK simulation data [29] and the NS equations with Stops' MFP model and second-order slip condition (24). In the slip flow regime, both the PL model and Stops' MFP based slip model agree reasonably well with the experimental data and BGK simulation results. However, around $\delta_{m} \sim 1$, Stops' MFP based slip model starts to significantly underpredict the flow rate, whilst the PL model follows the BGK simulation data reasonably well until $K n$ reaches about 10 . PL model results with an exponent $n$ value of 5 are also shown in Fig. 7a to illustrate the effects of $n$ on the flow rate in different flow regimes. The PL model with $n=5$ follows the PL model with $n=3$ until around $\delta_{m} \sim 1$, but then it starts to underpredict the flow rate, although reasonable agreement with the experimental data is still obtained in the free-molecular regime.

Flow rate results of our PL model with $n=3$ are shown in Fig. 7b to compare with the exact Boltzmann solution [27], DSMC data [30], R26 results [28], NS equations with first- and second-order slip and the PL model with first-order slip. Here
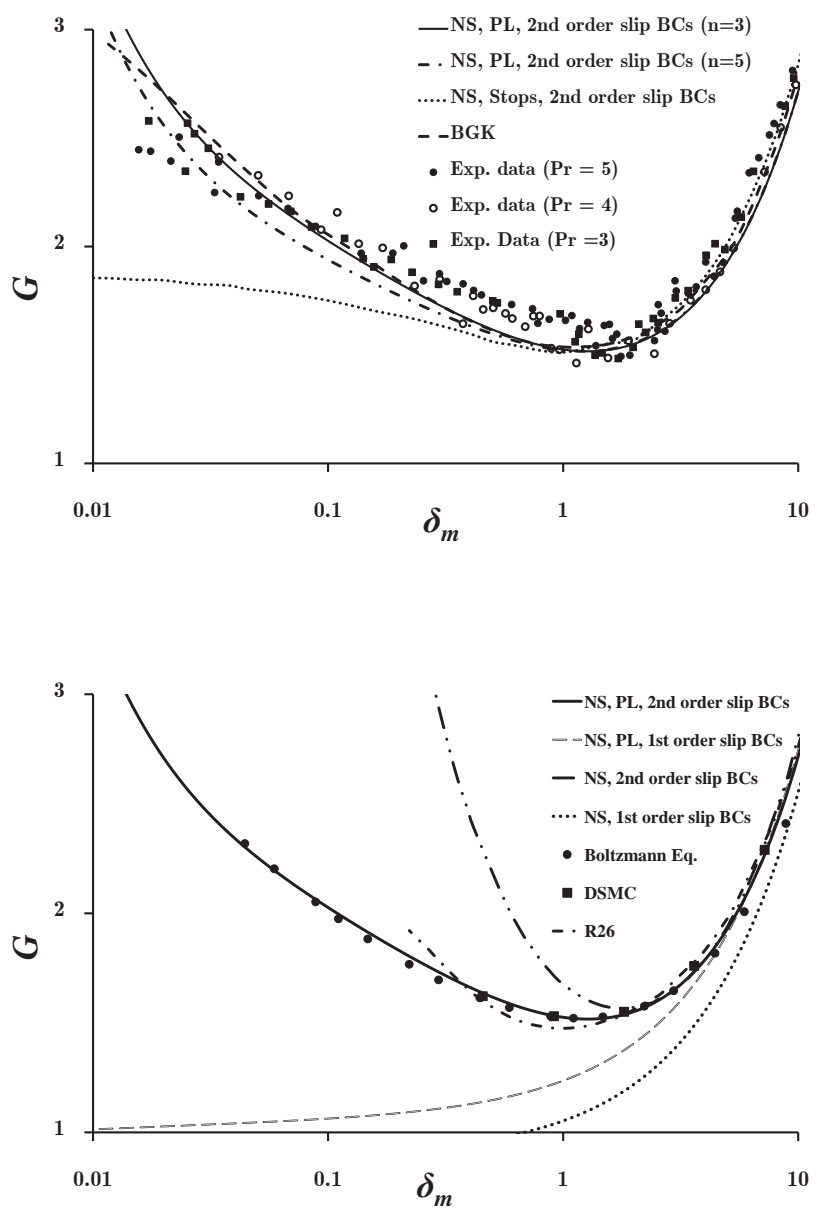

FIGURE 7. Normalized volume flow rate $(G)$ variation with inverse Knudsen number $\left(\delta_{m}\right)$. Comparison of power-law (PL) model results with: (a) experimental data [21] and BGK simulation results [29] (where $\operatorname{Pr}$ is the ratio of inlet to outlet pressure in the experiments); (b) DSMC data [30], the solution of the Boltzmann equation [27] and the conventional NS equations with first- and second-order slip.

the first-order slip for the PL based MFP NS equations results are obtained simply by setting $C_{2}=0$. For $K n>0.1$, which is just beyond the slip flow regime, the NS equations with first-order slip underpredict the flow rate, although the other hydrodynamic models are in good agreement with the Boltzmann and DSMC data up to $K n \sim 0.2$. The NS results with second-order slip are good up to $K n \sim 0.5$, but significantly overpredict beyond that. The R26 equations show good agreement until $K n \sim 2$, and our PL model with second-order slip follows the Boltzmann results up to $K n \sim 10$. Both PL and NS models with first-order slip fail to predict the Knudsen minimum. The other three hydro- 
dynamic models show a Knudsen minimum, however, the NS equations with second-order slip predict the minimum at a value of $K n$ smaller than that predicted by the Boltzmann equation.

\section{DISCUSSION AND CONCLUSIONS}

The key physics of the rarefied gas flow in the Knudsen layer has been outlined, and we have proposed an analysis based on a power-law (PL) probability distribution function for free-paths of molecules in a confined gas. The PL model is validated with molecular dynamics simulation data. A constitutive scaling approach to model the Knudsen layer within a continuum fluid dynamics framework has also been described. This has been tested for the case of isothermal pressure-driven gas flow through a microchannel. Our approach provides good results for both mean-free-path (MFP) and cross-sectional velocity profiles up to $K n \sim 1$, and for integral flow parameters such as flow rate up to $K n \sim 10$. Previous exponential probability distribution function MFP models, that assume thermodynamic equilibrium, provide accurate results only up to $K n \sim 0.2$, and normalized flow rate results are reasonable only up to $K n \sim 1$.

While the Navier-Stokes (NS) equations with higher-order slip boundary conditions can correctly capture the wall-slip by tuning the slip coefficients, they may not provide accurate velocity profiles in both the Knudsen layer and the bulk flow regions. The NS equations with an exponential-based MFP can provide good velocity profiles in the Knudsen layer up to the early transition regime. However, with our PL model we could influence the velocity profile at a specified Knudsen number through the exponent $n$.

An advantage of the PL MFP constitutive scaling technique is that it is based on simple physical arguments, which can successfully be implemented in conventional CFD codes, and is still able to capture some of the essential trends associated with the complex non-equilibrium physics of the Knudsen layer.

While it is important not to draw strong conclusions based on these simple test cases alone, the present results may motivate future work into understanding the origin of non-equilibrium physics in rarefied gases, incluing:

1. validation of the power-law probability distribution function for a range of flow systems geometries through molecular dynamics simulations;

2. non-equilibrium distribution functions are also a feature of rarefied gas shock waves. Power-law based diffusion coefficients [13] could also be modeled as a function of length scales defined in terms of local density and temperature gradients [3];

3. conventional higher-order velocity slip and temperature jump boundary conditions are derived under the assumption of constant MFP in Knudsen layers - derivations of these boundary conditions should be revisited based on geometry- dependent effective MFP models.

\section{ACKNOWLEDGMENT}

The authors would like to thank Erik Arlemark of the University of Strathclyde, UK, for providing molecular dynamics simulation data. The research leading to these results has received funding from the European Community's Seventh Framework Programme FP7/2007-2013 under grant agreement ITN GASMEMS no. 215504.

JMR would like to thank the Royal Society of Edinburgh (RSE) for support through a RSE/Scottish Government Support Research Fellowship.

\section{REFERENCES}

[1] Maxwell, J. C., 1879, On stresses in rarefied gases arising from inequalities of temperature, Philos. Trans. Roy. Soc., Part 1, 170, 231-256.

[2] Lockerby, D. A., Reese, J. M. and Gallis, M. A., 2005, The usefulness of higher-order constitutive relations for describing the Knudsen layer, Phys. Fluids, 17, 100609.

[3] Bird, G. A., 1994, Molecular Gas Dynamics and the Direct Simulation of Gas Flows, Oxford University Press, New York.

[4] Lockerby, D. A., Reese, J. M. and Gallis, M. A., 2005, Capturing the Knudsen layer in continuum-fluid models of nonequilibrium gas flows, AAIA J., 43, 1391-1393.

[5] O'Hare, L., Scanlon, T. J., Emerson, D. R. and Reese, J. M., 2008, Evaluating constitutive scaling models for application to compressible microflows, Int. J. Heat Mass Trans., 51, 1281-1292.

[6] Cercignani, C., Frangi, A., Lorenzani, S. and Vigna, B., 2007, BEM approaches and simplified kinetic models for the analysis of damping in deformable MEMS, Eng. Anal. Boundary Elem., 31, 451-457.

[7] Lilley, C. R. and Sader, J. E., 2007, Velocity gradient singulary and structure of the velocity profile in the Knudsen layer according to the Boltzmann equation, Phys. Rev. E, 76, 026315.

[8] Lockerby, D. A. and Reese, J. M., 2008, On the modelling of isothermal gas flows at the microscale, J. Fluid Mech., 604, 235-261.

[9] Stops, D. W., 1970, The mean free path of gas molecules in the transition regime, J. Phys. D: App. Phys., 3, 685-696.

[10] Arlemark, E. J., Dadzie, S. K., and Reese, J. M., 2010, An extension to the Navier-Stokes equations to incorporate gas molecular collisions with boundaries, J. Heat Transf. (Trans. ASME), 132, 041006.

[11] Guo, Z. L., Shi, B. C., and Zheng, C. G., 2007, An extended Navier-Stokes formulation for gas flows in the Knudsen layer near a wall, Europhys. Lett., 80 (2), 24001-24006. 
[12] Arlemark, E. J. and Reese, J. M., 2009, Investigating the effect of solid boundaries on the gas molecular mean-freepath, ASME ICNMM2009-82080, Pohang, South Korea.

[13] Fa, K. S. and Lenzi, E. K., 2003, Power law diffusion coefficient and anomalous diffusion: analysis of solutions and first passage time, Phys. Rev. E, 67, 061105.

[14] Montroll, E. W. and Scher, H., 1973, Random walks on lattices. IV. Continuous-time walks and influence of absorbing boundaries, J. Stat. Phys., 9 (2), 101-135.

[15] Bird, R. B., Stewart, W. E. and Lightfoot, E. N., 1960, Transport Phenomena, John Wiley and Sons (WIE), New York.

[16] Yasuhiko S., Haruka K., Kazuki M. and Hiroyuki T., 2004, Two-particle diffusion and fine-scale structures of turbulence, Proceedings of the IUTAM Symposium on Elementary Vortices and Coherent Structures: Significance in Turbulence Dynamics, Kyoto, Japan.

[17] Sone, Y., 2002, Kinetic Theory and Fluid Dynamics, Birkhauser, Boston.

[18] Cercignani, C., 1990, Mathematical Methods in Kinetic Theory, Plenum Press, New York.

[19] Grolimund, D., Borkovec, M., Federer, P. and Sticher, H., 1995, Measurement of sorption isotherms with flowthrough reactors, Environ. Sci. Techno., 29, 2317-2321.

[20] Cercignani, C., 1988, The Boltzmann Equation and its Applications, Springer-Verlag, New York.

[21] Ewart, T., Perrier, P., Graur, I. A. and Meolans, J. B., 2007, Mass flow rate measurements in a microchannel, from hydrodynamic to near free molecular regimes, J. Fluid Mech., 584, 337-356.

[22] Dongari, N., Agrawal, A. and Agrawal, A., 2007, Analytical solution of gaseous slip flow in long microchannels, Int. J. Heat Mass Trans., 50, 3411-3421.

[23] Reese, J. M. and Zhang, Y. H., 2009, Simulating fluid flows in micro and nano devices: the challenge of nonequilibrium behaviour, J. Comp. Theor. Nanosci., 14, 2061 2074.

[24] Deissler, R.G., 1964, An analysis of second-order slip flow and temperature jump boundary conditions for rarefied gases, Int. J. Heat Mass Trans., 7, 681-694.

[25] Cercignani, C., 1964, Higher order slip according to the linearized Boltzmann equation, Institute of Engineering Research Report AS-64-19, University of California, Berkeley.

[26] Hadjiconstantinou, N. G., 2003, Comment on Cercignani's second-order slip coefficient, Phys. Fluids, 15(8), 23522355.

[27] Ohwada, T., Sone, Y. and Aoki, K., 1989, Numerical analysis of the Poiseuille and thermal transpiration flows between parallel plates on the basis of the Boltzmann equation for hard-sphere molecules, Phys. Fluids A 1, 2042-2049.

[28] Gu, X.-J. and Emerson, D. R., 2009, A high-order moment approach for capturing non-equilibrium phenomena in the transition regime, J. Fluid Mech., 636, 177-226.

[29] Loyalka, S. K., 1975, Kinetic theory of thermal transpiration and mechanocaloric effects II, J. Chem. Phys., 63, 4054-4560.

[30] Hadjiconstantinou, N.G., 2006, The limits of Navier-Stokes theory and kinetic extensions for describing small scale gaseous hydrodynamics, Phys. Fluids, 18, 111301. 